The relative amplitudes of the first ground-pulse $G$ and its reflections $1,2,3,4$ and 5 alter noticeably with distance. At moderate distances, as would be expected, $G$ is the largest $(A, B, C)$. Farther out $(D, E)$, attenuation of the ground-wave reduces its amplitude below that of the first sky wave. Still farther away from the parent storm $(F)$ the first sky wave must graze the earth before reaching the receiver and becomes attenuated from this cause also, so that the second sky wave has the maximum amplitude.

The atmospherics which exhibit the clear evidence of reflection here discussed are all due to flashes to ground. As has been pointed out elsewhere, discharges within the thundercloud produce effects which are weaker in intensity and more complex in form.

We have to thank Mr. I. Katz, Prof. D. B. Hodges and Mr. Phillips of Howard College, Durban, and Mr. R. A. Jubb of the Meteorological Department, Southern Rhodesia, for operating the directionfinders used in locating thunderstorms.

B. F. J. Schonland.

J. S. ELDER.

J. W. VAN WYK.

G. A. Cruickshank.

Bernard Price Institute,

University of the Witwatersrand, Johannesburg.

Watson Watt, R. A., Herd, F. F., and Lutkin, F. E., Proc. Roy. Soc., A, 162, 267 (1937). Appleton, E. V., and Chapman, F. W. Proc. Roy Soc. A 159. Appleton, L. Nickson, A and Webster, H., NATUR, '139, 837 (1937), Laby. T. H., Nicholls, F. G., Nickson, A. F. B., and McNeill, J. J.,

$$
\begin{aligned}
& \text { T. H., Nicholls, F. G., Nick } \\
& \text { NATURE, 142, } 353 \text { (1938). }
\end{aligned}
$$

\section{Disintegration of Nitrogen by Fast Neutrons}

According to researches published up to the present time, bombarding nitrogen with fast neutrons produces the two following nuclear reactions :

(i) ${ }^{14} \mathrm{~N}+{ }^{1} n={ }^{11} \mathrm{~B}+{ }^{4} \mathrm{He}$

(ii) ${ }^{14} \mathrm{~N}+{ }^{1} n={ }^{14} \mathrm{C}+{ }^{1} \mathrm{H}$.

The present paper deals with the determination of the cross-sections $\sigma$ for these two processes and the total energy $Q$ evolved in reaction (i).

An ionization chamber filled with nitrogen was bombarded with fast neutrons obtained by the reaction ${ }^{2} \mathrm{D}\left({ }^{2} \mathrm{D},{ }^{1} n\right)^{3} \mathrm{He}$. The products of transformation were detected with the help of a linear amplifier and of an oscillograph. Both neutron source and amplifier have been described in detail in a former paper'.

In the accompanying figures are plotted two statistics for the process ${ }^{14} \mathrm{~N}\left({ }^{1} n,{ }^{4} \alpha\right)^{11} \mathrm{~B}$. Curve $A$ corresponds to an angle of observation of $0^{\circ}$ (neutronic energy $2.8 \mathrm{Mev}$.) and Curve $B$ to an angle of $90^{\circ}$ (neutronic energy $2 \cdot 36 \mathrm{Mev}$.). By 'angle of observation' we understand the angle between the direction of the incident deuterons and the direction of observation of the emitted neutrons. The crosssection $\sigma$ and the reaction energy $Q$ for the process ${ }^{14} \mathrm{~N}\left({ }^{1} n,{ }^{4} \alpha\right){ }^{11} \mathrm{~B}$ can be determined, since the intensity of the neutron source and the energy of the emitted neutrons are known. The study of a thousand of such processes gave the following results:

$$
\sigma=1 \cdot 63 \times 10^{-25} \mathrm{~cm} .{ }^{2} . Q=-0.43 \mathrm{Mev} .
$$

From corresponding measurements for the process ${ }^{14} \mathrm{~N}\left({ }^{1} n,{ }^{1} \mathrm{H}\right){ }^{14} \mathrm{C}$ we found a cross-section equal to $\sigma=0.4 \times 10^{-25} \mathrm{~cm} .^{2}$.

The difference between the neutronic energies corresponding to the two angles $0^{\circ}$ and $90^{\circ}$ can be
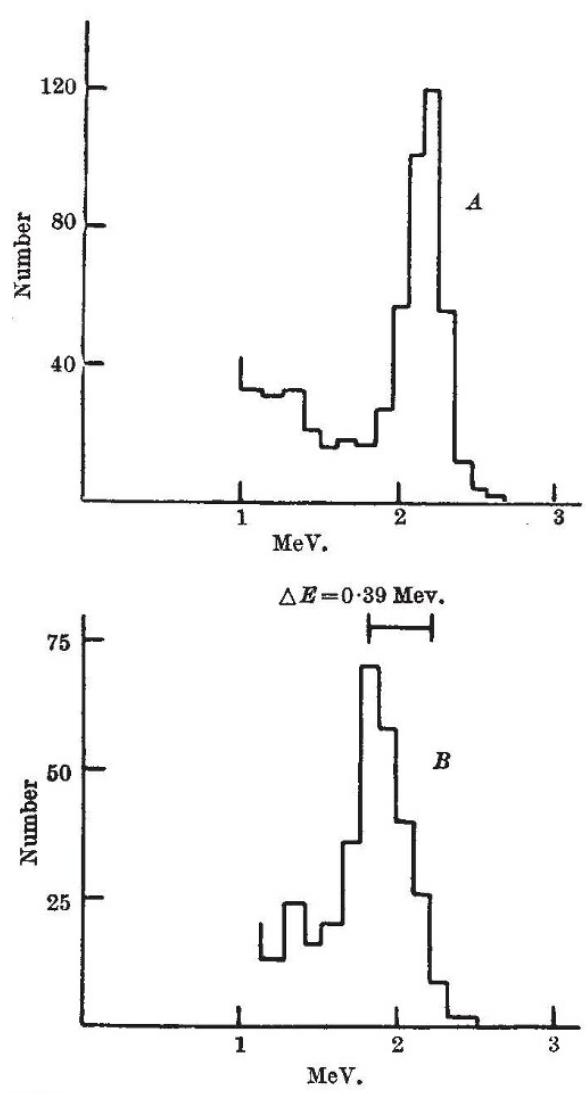

OSCILLOGRAPH DEFLECTIONS AS A FUNCTION OF THE CORRESPONDING ENERGY

calculated by means of the theorems of conservation of energy and momentum. If the deuterons are accelerated by $140 \mathrm{kv} .$, this energy difference is equal to $0.44 \mathrm{Mev}$. The observed difference, $0.39 \mathrm{Mev}$., is, within the limits of possible errors, in agreement with the calculated value.

A complete account of the experiments will be published in the Helv. phys. Acta.

Physical Institute,

E. BALDINGER.

Federal Technical College, Zurich.

April 1.

${ }^{1}$ Baldinger, Huber and Staub, Helv. phys. Acta, 11, 245 (1938).

\section{Crystal Structure of Glucosamine Hydrobromide}

Progress in the detailed analysis of carbohydrate crystal structures has hitherto been slow, on account of the lack of guidance from physical (optical and magnetic) properties of the crystals, the wide choice of molecular conformations on which 'trial and error' calculations might be based, the absence of outstandingly strong reflection intensities, and the asymmetry of the compounds concerned. The isomorphism of $\alpha$-chitosamine hydrochloride and hydrobromide has now enabled us to determine the positions of all the atoms (other than hydrogen) in the crystal lattices of these substances by synthetic methods alone, no stereochemical assumptions being necessary. 